\title{
Morphological Applications for Maps Construction in Path Planning Tasks
}

\author{
J. Azorín ${ }^{1}$, G. De Miguel ${ }^{1}$, F.A. Pujol ${ }^{1}$, J.M. García ${ }^{1}$, M. Pujol $^{2}$, and R. Rizo ${ }^{2}$ \\ 1 Depto. Tecnología Informática y Computación, \\ Universidad de Alicante Ap. Correos 99, 03080 Alicante, España \\ \{jazorin, demiguel, fpujol, juanma\}@dtic.ua.es, \\ 2 Depto. Ciencia de la Computación e Inteligencia Artificial \\ Universidad de Alicante Ap. Correos 99, 03080 Alicante, España \\ \{mar, rizo\}@dccia.ua.es
}

\begin{abstract}
The process of taking decisions in an autonomous system has a strong dependence on the information received from the environment in which it works. The path planning process for a mobile robot is a subset of the problems that have to be solved in an automatic system of decisions, where the appropriateness of the method is roughly constrained not only by the achievement of a well-suited information from the environment, but also by how this information is acquired. The main objective of this work is to describe a robust model for robotic path planning in unknown environments. The proposed general model is based on obtaining secure paths where the robot will be able to move so as to complete its task. Thus, from an unknown location, the required information is collected by employing a mobile robot that moves freely through a real world with some generic behaviors that let the robot explore the world.
\end{abstract}

\section{Introduction}

The growing incorporation of robots to our environment [1] shows the emergent proposal of systems that have an intelligent behavior and, as a result, makes it possible to have a great amount of technical solutions that improve the execution of specialized tasks.

From an Artificial Intelligence point of view, the robotic systems have also an enormous interest when the research is focused on the associated processes that intelligence involves, where intelligence refers to an essential feature in some complex systems, such as those ones who involve learning and autonomous control [2]. This way, an autonomous robot can be considered as an embedded system and, in general, as an entity with some reactive and deliberative abilities with adaptive behavior; consequently, it is related to dynamic environments.

The multidisciplinary research in Robotics includes many different approaches in the methods for designing a robot. First, we have the classical point of view that utilizes a three-level architecture: Functional Level, Executive Level and Planning Level, as it can be seen in [3]. On the other hand, a more flexible

A. Sanfeliu and J. Ruiz-Shulcloper (Eds.): CIARP 2003, LNCS 2905, pp. 651-658 2003.

(C) Springer-Verlag Berlin Heidelberg 2003 
characterization is based on some analogies with biological evolution [4]. As a consequence, the first approach implies that these architectures must be simplified quite often since many requests have to be completed at the same time. On the contrary, the second contribution provides a specification based on behaviors that are modelled by means of complex control algorithms that cover a greater range of requests.

Nevertheless, most of the projects proposed for robotic prototypes include architectures based on general criteria of designing in order to solve real-world problems, and also incorporate special features for its adaptation to special environments [5], 6].

In this work, we propose a general model for the recognition of the world in which a robot interacts, in order to follow a collision-free path within the environment. First, we need to define the fundamental concepts for the characterization of the environment and the acquisition of information, as it is described in Section 2. After that, a robust model to generate a map, considering some topological landmarks, is introduced in Section 3. Then, Section 4 explains how the received information is processed and, subsequently, in Section 5 the experimentation is widely illustrated to verify the accomplishment of our research objectives. Finally, we conclude with some important remarks in Section 6 .

\section{Design of a Robust Model}

Let us consider a general approach to the situation shown in the previous section. First of all, the problem can be described as the obtaining of safe paths where a mobile robot would be able to wander with no collision, in order to fulfill some objectives. In relation to this, from an unknown world, the robot will obtain information that, once processed, will lead to obstacle-free paths.

To do this, we need to establish theoretically the representation of the factors that would interact with the robot. Since our purpose is obtaining an interpretation function that approximates the environment as good as possible, the suitability of the solution will be mainly determined by the quality of that representation. That is, we want to define a function that synthesizes an image from the perception of the environment; as it is described in the following sections, the synthesization uses Mathematical Morphology methods.

Thus, the algorithm that builds maps of the environment can be divided into a series of independent steps, where each step of lower level provides the necessary information for the upper level processes. This way, it can be considered at the same time as an abstraction of the immediately lower levels.

As a result, this model gives a multi-level architecture that can be divided into the following layers:

- Acquisition of information

- Positional information

- Topological information

- Constraints of the environment 
- Information processing

- Generation of knowledge

Next, a more detailed description of these levels will be found.

\section{Acquisition of Information}

As one would expect, the data acquisition depends both on the environment and on a set of features that define the operation of the mobile robot. There are two kind of features:

- The internal sensing capabilities, which are related to obtain the robot location in a real world.

- The external sensing capabilities to acquire information by interacting with the environment (laser, sonar, cameras,... ).

In the following sections these features are explained.

\subsection{Positional and Topological Information}

Considering the internal capabilities, the positional information will be determined mainly by the actual position of the robot in a workspace. Of course, the robot's self-knowledge about what is its location also has a great effect on the final result.

On the other hand, the external capabilities can be calculated by acquiring topological information; these data are extracted from the environment where the robot is interacting to. Thus, the topological landmarks are related to the different zones in which an environment can be divided. Therefore, each of these zones must be located in the world maps and, as a result, the landmarks will establish a possible positioning state of the robot. Let $e_{i}$ be all the possible states.

To do this, in our design we will consider that the robot includes a sonar sensor ring, as reading these sensors will give the system enough knowledge to distinguish the topological zones in which a map is divided. Then, the likeness function should be defined; starting from a group of sonar readings, this function provides the probability that the sonar measurements have been created from each one of the states $e_{i}$. The likeness function is determined by:

$$
v\left(r \mid e_{i}\right)=\exp \left(-\operatorname{dist}\left(r, e_{i}\right) / a\right) .
$$

where $a$ is a correction factor, such that $a \geq 1$, and dist is the euclidean distance between the current sensor reading $r=\left(r_{1}, r_{2}, \ldots, r_{n}\right)$ and the representation of each state $e_{i}$ in the database:

$$
\operatorname{dist}\left(r, e_{i}\right)=\sqrt{\sum_{j=1}^{n}\left(r_{j}-e_{i_{j}}\right)^{2}} .
$$


It is also necessary to establish a model for the robot's movement, which indicates the probability of either changing from a state to another one or keeping on the same state:

$$
m\left(e_{i} \mid e_{j}\right)
$$

This model has been implemented by using an FSM (Finite State Machine), so that the probability of a transition between states must be estimated according to the environment.

Finally, the acquisition function should be tested. This point can be completed as the robot follows a path, while it avoids obstacles with a constant velocity. Then, we define a probability function $c_{t}\left(e_{j} \mid r\right)$ in order to keep the robot on the same state or to change it, if necessary, when the movement is produced:

$$
c_{t}\left(e_{j} \mid r\right)=\alpha v\left(r \mid e_{j}\right) \sum_{i=1}^{n} m\left(e_{j} \mid e_{i}\right) c_{t-1}\left(e_{i} \mid r\right) .
$$

where $\alpha$ is a real constant.

\subsection{Topological Landmarks}

From the former discussion, it is clear that each landmark will identify a locating state $\left(e_{1}, e_{2}, \ldots, e_{n}\right)$. Let us consider the environment defined in Fig. 11. In this case, the possible landmarks are:

- Corridor: e1

- Corner: e2

- T: e3

- Crossing: e4

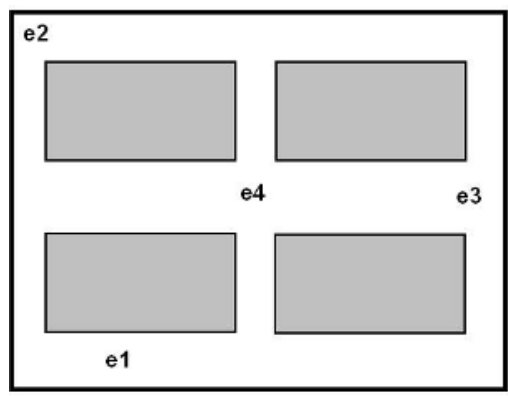

Fig. 1. Proposal of environment

Therefore, in this workplace we can find the four landmarks depicted in Fig. 2. 

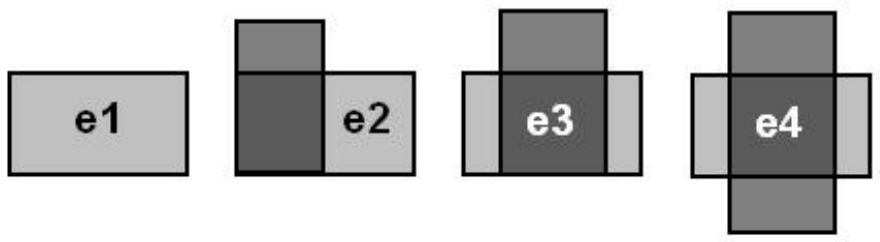

Fig. 2. The four topological landmarks

The experimentation is arranged by means of a robot that includes 24 sonar sensors. Then, each reading from the sensors is computed and, afterwards, the robot training is carried out for each landmark that has been previously identified in the map. To perform this, the robot is placed at each landmark and all the possible readings are obtained. From that information, an image of the environment can be created and, as a result, a map is generated; this is explained in the next section.

\section{Information Processing and Generation of Knowledge}

The next level in the architecture performs a data processing after that the information is received at the lower level. As we have pointed out before, the collected data come from a group of sonar sensors which provide a set of points (i.e., a reading) that makes a representation of the environment.

In order to achieve a reliable image starting from this set of readings, we propose to use a morphological dilation of the obtained points to extract an estimated map (see Fig. 3). Then, the dilation is carried out considering both the robot architecture as well as its physical dimensions. The objective is to find a set of points that provides free paths for a no-collision navigation and, as a result, a synthesized image that contains a map of the environment.

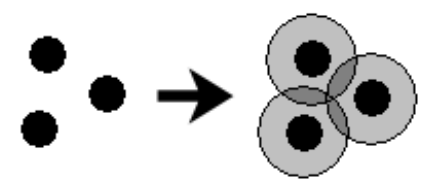

Fig. 3. Dilation of a sensor reading

It would be very important to take into account the obtained positional and topological information so that the dilation could create separate regions for the different landmarks and, consequently, could provide a better data representation for the path planning process (Fig. (4)). 


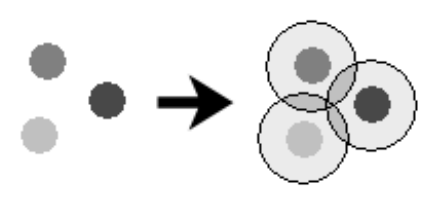

Fig. 4. Dilation of points considering topological information

Finally, the highest level includes the generation of an image from the set of regions that are labelled according to the landmarks that exist throughout the environment. This process can also be completed by means of the dilation of the sensor reading points. In the following section we describe some examples of the application of these operations.

\section{Experiments}

Let us consider now the results of some experiments completed for our model. Thus, the tests have been simulated and, afterwards, the robot is supposed to work in the environment built in Fig. 1 As shown before, this world consists of a set of points labelled as corridor, corner, T and crossing points (see Fig. 5 (a)).

Let us assume that the robot moves along a path established by a generic pattern of movement. Then, the positional and topological information is extracted while acquiring points in a planned trajectory, as shown in Fig. 5 (b).

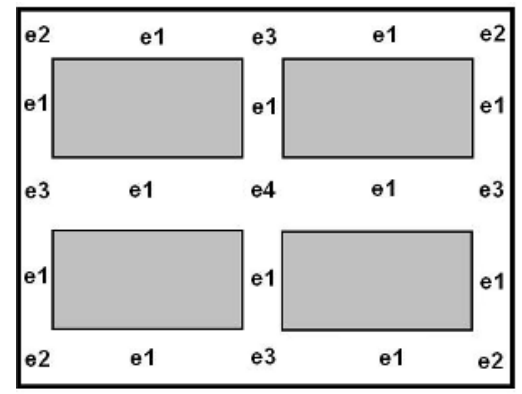

(a)

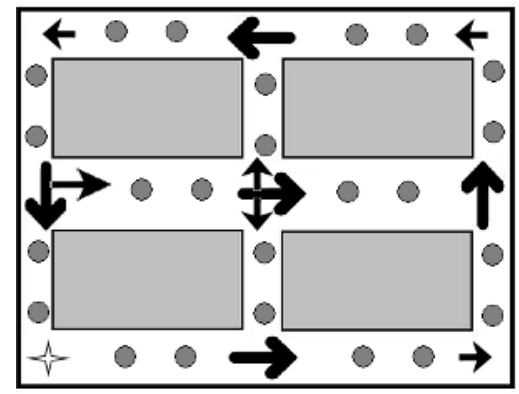

(b)

Fig. 5. (a) Landmark labelling for an environment. (b) Sample path for the considered environment

This way, when the mobile platform goes along a path, the acquisition of information level provides a series of points where the robot is able to move (see Fig. 6).

As the robot receives the data, the information processing system synthesizes the map of the environment in real time, considering every set of points received. 

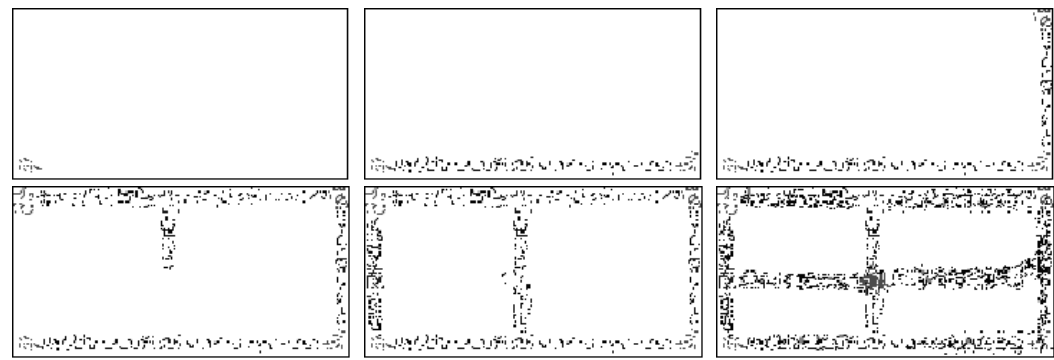

Fig. 6. Results of the acquisition level

Consequently, once the system has labelled the points according to one of the possible landmarks, the morphological dilation provides the regions where an object collision has a low probability (Fig. 7).
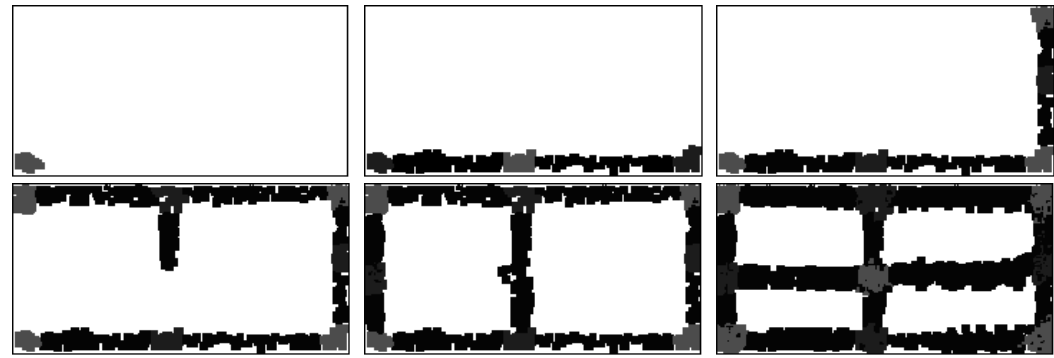

Fig. 7. Results of the information processing level

The last images show that the synthesis is completed with an iterative method, where all the information that the sensors provide in real time allows that the map of the environment has a good quality without consuming many resources. As we can see, the main goals of our research task have been accomplished.

\section{Conclusions}

In general terms, the path planning process for a mobile robot is strongly influenced by the precision of the acquisition process. Thus, it can be modified both by the quality of the information obtained from the environment, and the attributes of the system and the environment in which it works.

In this paper, we have developed a proposal of a model for the generation of a map in unknown environments; then we have designed an information acquisition process that allows a representation of the environment for the path planning 
process. The experimentation shows that the prototype is quite robust and could be applied in some indoor environments.

As a future work, it would be so desirable the application of the model in a real environment, as well as consider new simulation experiments with different environments. This will lead to a more accurate designing method so that the robot internal hardware could be efficiently implemented. As a result, it can support all the possible requests that the model would have in a real situation and, finally, the function of acquisition can be generalized for other sensing models. Therefore, we can achieve more robustness in the positional and topological acquisition of information and, consequently, in the higher levels of the architecture.

Acknowledgements. This work has been supported by the Spanish Oficina de Ciencia y Tecnología (OCYT) of the Generalitat Valenciana, project number CTIDIA/2002/183 and by the Spanish Comisión Interministerial de Ciencia y Tecnología (CICYT), project number TIC2001-0245-C02-02.

\section{References}

1. Parker, L. E.: Lifelong adaptation in heterogeneous multi-robot teams: response to continual variation in individual robot performance. Autonomous Robots, 8 (2000) 239-267

2. Brooks, R.A.: Intelligence without reason. In Proc. of the 12th International Joint Conference on Artificial Intelligence, Sydney, Australia, (1991) 569-595

3. Tzafestas, E.S.: Reactive robots in the service of production management, Journal of Intelligent and Robotic Systems, 21 (1998) 179-191

4. Arkin, R.C.: Behavior-based robotics. MIT Press (1998)

5. Zomaya, A.Y., Wright, M.R., Nabham, T.M.: On the path planning problem in the vicinity of obstacles. Cybernetics \& Systems, 29 (1998) 807-868

6. Pujol, F., García Chamizo, J. M., Fuster, A., Pujol, M., Rizo, R.: Use of mathematical morphology in real-time path planning. Kybernetes, 31 (2002) 115-123 